\title{
El aprendizaje significativo. Algunas consideraciones para su alcance en el Instituto Superior Tecnológico Almirante Illingworth
}

\section{The significant learning. Some considerations for their reach in the Institute Superior Technological Admiral Illingworth}

\author{
MSc, Laura Yazmín Marín Granados ${ }^{1}$ \\ Imarin@aitec.edu.ec \\ https//orcid.org/0000-0003-1106-7721
}

\begin{abstract}
RESUMEN
La educación actual exige la formación de un hombre nuevo, pensante, reflexivo, analítico y es por ello que las instituciones de educación superior establecen un modelo pedagógico que permita alcanzar estos resultados. Para ello es importante desarrollar en los estudiantes aprendizajes significativos, los cuales se generan cuando el aprendiz interactúa con el medio que lo rodea y en este proceso construye representaciones personales desde su estructura cognitiva que les permitirá su aplicación en escenarios reales de su práctica profesional. Se realiza una investigación exploratoria de tipo cualitativo, para recabar información empírica y documental sobre la situación de las aulas en cuanto al aprendizaje significativo, encontrando que son pocas las actividades que se planifican en función de este objetivo y que los docentes no logran aplicar en todas sus clases estrategias que generen aprendizajes diferentes. Se proponen estrategias de enseñanza, teniendo en cuenta el objetivo a alcanzar, como eslabón fundamental para el desarrollo de aprendizajes significativos, las cuales orientan y direccionan el accionar del docente, teniendo en cuenta las características, estilos de aprendizaje y necesidades individuales de cada uno de los estudiantes.
\end{abstract}

Recibido: 1/4/2020, Aceptado: 1/6/ 2020

Palabras clave: Aprendizaje, aprendizaje significativo, estrategias de enseñanza, modelo pedagógico, estructura cognitiva

\section{ABSTRACT}

Current education requires the formation of a new, thinking, reflective, analytical man and that is why higher education institutions establish a pedagogical model that allows achieving these results. For this, it is important to develop significant learning in students, which is generated when the learner interacts with the environment that surrounds him and, in this process, builds personal representations from his cognitive structure that will allow their application in real scenarios of his professional practice. A qualitative exploratory research is carried out to gather empirical and documentary information on the situation of the classrooms in terms of meaningful learning, finding

\footnotetext{
${ }^{1}$ Instituto Superior Tecnológico Almirante Illingworth, Guayaquil, Ecuador.
} 


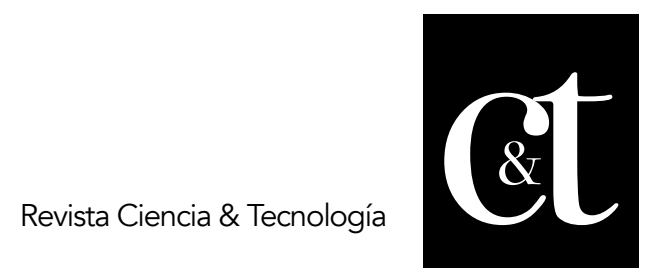

No. 27, 31 de julio de 2020

ISSN impreso: 1390 - 6321

ISSN online: 2661 - 6734

that few activities are planned based on this objective and that teachers are not able to apply in all their classes strategies that generate different learning. Teaching strategies are proposed, taking into account the objective to be achieved, as a fundamental link for the development of meaningful learning, which guide and direct the actions of the teacher, taking into account the characteristics, learning styles and individual needs of each of the students.

Keywords: Learning, meaningful learning, teaching strategies, pedagogical model, cognitive structure

\section{Introducción}

El cambio educativo constituye realidad y premisa para el Ministerio de Educación Superior del Ecuador, este cambio se debe asumir desde una perspectiva desarrolladora y que debe tener en cuenta la búsqueda de acciones concretas para alcanzar la excelencia académica, viendo contexto local, regional y nacional de educación. Estos cambios deben aparejarse de un esfuerzo por cada institución en alcanzar calidad educativa desde estrategias inteligentes que permitirán a los futuros profesionales enfrentar los desafíos y problemas dentro de su contexto profesional.

El aprendizaje desde el nivel cognitivo constituye criterio de análisis para varias ciencias. Psicólogos y pedagogos vienen investigando cómo potenciar un aprendizaje significativo, aunque falta por hacer y desde el campo científico - investigativo. Ante esta premisa se hace necesario definir algunos términos que esclarecerán el rol del estudiante ante la actitud y conducta para aprender y el del docente desde sus estrategias para que se logre el aprendizaje significativo (Varela, 2018).

Es importarte partir de algunas interrogantes que le permitirán al docente entrar en un proceso de reflexión necesaria para conocer a su estudiante y la forma en que maneja o potencia su inteligencia, ¿cómo cada estudiante procesa la información que recibe? sobre este procesamiento ¿cómo el docente, la institución contribuye a la formación y desarrollo de un procesamiento eficiente? Es importante que se reflexione en cuestiones importantes que define la educación superior y que el instituto establece en cada proyecto de carrera, y es el hecho de qué estudiante que aspiramos a formar, que habilidades, destrezas y competencias deben haber alcanzado al final de este proceso académico, aspectos que se definen claramente en el perfil de egreso de las carreras, criterios que se consideran necesarios asumir para un aprendizaje significativo.

Se parte de estos criterios por la necesidad de analizar la actuación y conducta, ya que desde sus propias definiciones se puede asumir que la conducta hace referencia al plano externo (lo observable), por su parte la actuación puede valorarse desde el plano interno propio del sujeto. Desde esta concepción se asume que la actuación inteligente constituye la forma en que el estudiante adquiere y procesa la información, tanto la previa almacenada como aprendizaje ya adquirido y la nueva que se trabajará, realizando un proceso de vinculación cognitivo entre lo viejo aprendido y lo nuevo por aprender, criterio que está fundamentado en el modelo pedagógico definido por la institución objeto de estudio. 


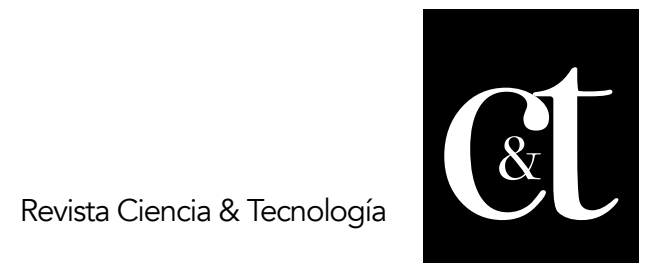

No. 27, 31 de julio de 2020

ISSN impreso: 1390 - 6321

ISSN online: 2661 - 6734

Para el aprendizaje significativo el estudiante debe partir de este proceso de relación, ya que será desarrollador, significativo lo que aprenda cuando sea capaz de desarrollar estrategias cognitivas que garanticen la propuesta de acciones de solución ante diversos problemas que se presenten en su determinado contexto de actuación profesional.

\section{Desarrollo}

Al abordar aspectos básicos sobre la calidad de la educación se debe partir de las acciones de la institución hacia el desarrollo del potencial cognitivo de sus estudiantes, hacia aprendizajes desarrolladores y significativos, aspectos que le permitirán insertase de manera plena en su vida profesional, social, así como la capacidad que debe desarrollar para dar respuesta o soluciones rápidas a situaciones que se presenten en su contexto de actuación. Ante esta necesidad juega un rol importante el docente, ya que debe asegurar desde los diferentes componentes de aprendizaje brindar una adecuada atención a la diversidad educativa, ya que el docente direcciona sus acciones o estrategias metodológicas hacia propiciar en sus educandos este aprendizaje significativo.

Estudios anteriores han demostrado que la caracterización de los procesos de aprendizaje de los estudiantes sería el punto de partida para identificar las dificultades más significativas para asumir las dimensiones de un aprendizaje significativo (Zambrano, 2016). Estos estudios han concluido demostrando que las transformaciones debían centrarse en los aspectos relativos a la activación regulación del aprendizaje, a su significatividad, y a la motivación para aprender, es decir, definidos desde diferentes focos:

Se coincide con (Castellano, Reinoso, \& García, 2014) cuando establecen los diferentes focos que se deben tener en cuenta para trabajar desde los salones de clases este aprendizaje, los mismos que se definen a continuación:

- Foco en la activación y regulación del propio aprendizaje: otorgando especial atención a la creación de ambientes de aprendizaje productivos, creativos, meta cognitivos y cooperativos, en los que los estudiantes tengan la oportunidad y la necesidad, de participar activamente en la construcción de los conocimientos, de reflexionar acerca de los procesos que llevan al dominio de los mismos, de conocerse a sí mismos y a sus compañeros como aprendices, y de asumir progresivamente la dirección y el control de su propio aprendizaje.

- Foco de la significatividad: propiciará aprendizajes que permitan descubrir los vínculos esenciales entre sus contenidos, y que hagan de la búsqueda del sentido personal de los mismos la clave para la comprensión, para la conciencia de su utilidad (individual y social) y para su inserción en el proceso de desarrollo de la personalidad.

- Foco de las motivaciones intrínsecas del aprendizaje: se aprovecha el existente sistema de incentivos y motivos extrínsecos que subyacen en las actitudes positivas hacia la institución con vistas a satisfacerlas.

- Foco de las autovaloraciones que los sujetos tienen de sí mismos como aprendices: apoya a los estudiantes en la tarea del autoconocimiento objetivo, en la formación de una autoestima positiva, y en el establecimiento de metas, objetivos, y aspiraciones adecuadas que fomenten la necesidad de realizar aprendizajes permanentes y la seguridad de tener la preparación para ello.

Son diversos los autores que han definidos los pasos o procedimientos a seguir para trabajar el aprendizaje significativo, por su parte (González, 2018) refiere que los docentes deben partir de cuestionarse desde diversas interrogantes que les permita 


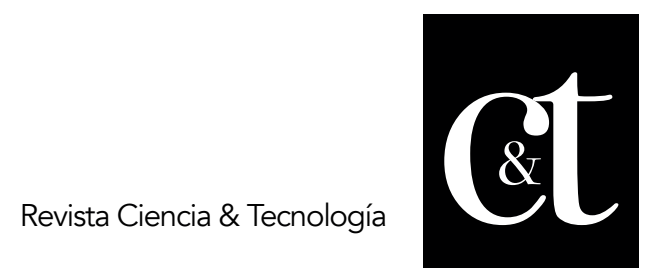

No. 27, 31 de julio de 2020

ISSN impreso: 1390 - 6321

ISSN online: 2661 - 6734

una reflexión, durante este proceso reflexivo el docente debe ir valorando las posibilidades que tienen sus estudiantes de desarrollar un aprendizaje activo y autorregulado, criterios que son sustentados en la dimensión activación - regulación que se define dentro de un aprendizaje significativo. Dentro de las preguntas que define como punto de partida del docente están:

- ¿Qué conocen mis estudiantes, y con qué profundidad lo conocen?

- ¿Cómo se apropiaron de este conocimiento? ¿Qué posición adoptan ante él? ¿Son simples receptores de información? ¿Son capaces de buscarla, producirla, pensar críticamente acerca de la misma?

- ¿Qué son capaces de hacer con ese conocimiento?

- ¿Qué conocen acerca de su propio aprendizaje? ¿Son capaces de reflexionar acerca del mismo?

- ¿Cómo se implican en este proceso? ¿Se sienten responsables del mismo?

- ¿Son capaces de controlar su aprendizaje, identificando cuáles son sus objetivos, auto-observándose, autoevaluándose, etc.?

- ¿Conocen cómo pueden mejorar su aprendizaje, hacerlo más eficiente?

(López, 2011) indica que el docente debe concebir estrategias de aprendizaje que permitan la activación y regulación del aprendizaje. Bajo el término estrategias se hace referencia a la vinculación de los procesos de pensamiento y aprendizaje, que suelen agrupar aspectos de muy diversa índole, desde habilidades y procedimientos motores hasta procedimientos cognitivos de naturaleza funcional superior, como los englobados por el rubro de meta cognición. Desde esta premisa las estrategias comprenden un plan diseñado con el objetivo de alcanzar una meta determinada, a través de un conjunto de acciones (que puede ser más o menos amplio, más o menos compleja) que se ejecutan controladamente. Existen estrategias sencillas, que permiten alcanzar objetivos a corto plazo. Otras requerirán el despliegue de acciones más complejas para resultados que son alcanzables solo a largo plazo (Palma-Orozco, García-Leyva, \& Ruiz-Ledesma (2020).

La enseñanza actual reconoce la concepción de que trabajar en los salones de clases un aprendizaje activo implica por parte del docente obligatoriamente la planificación, ejecución y evaluación de estrategias efectivas para alcanzar el aprendizaje. Es por ello que, las estrategias de aprendizaje serán acciones, tareas estructuradas desde conocimientos y procedimientos que los estudiantes deben alcanzar durante el módulo de cada asignatura. En su planificación el docente revisa el conjunto de procesos, acciones y actividades desde diferentes componentes de aprendizajes definidos en el Reglamento del Régimen Académico y que los estudiantes deben cumplir conscientemente para alcanzar un aprendizaje significativo. El planificar de manera eficiente las estrategias de enseñanza dependerá de las actividades y resultados de aprendizaje que hayan quedado establecidos en los diferentes proyectos de carrera.

Las estrategias de enseñanza aplicadas por el docente constituyen un eslabón importante para alcanzar el aprendizaje significativo, como ya se ha mencionado es un 


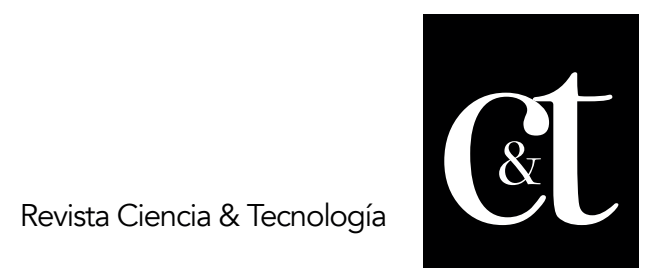

No. 27, 31 de julio de 2020

ISSN impreso: 1390 - 6321

ISSN online: 2661 - 6734

proceso donde los roles fundamentales se centran en el estudiante y su estilo de aprendizaje y en el docente y sus estrategias de enseñanza. Ausubel es el creador de esta teoría de aprendizaje significativo, término con significatividad cuando se aplica un modelo de enseñanza constructivista, esta teoría da una respuesta reveladora a la concepción que se debe asumir para alcanzar el aprendizaje, cuando este se encamine hacia la interacción del estudiante entre lo que aprende y el contexto donde debe aplicar lo aprendido. Para ello se debe partir de que el aprendizaje debe asumirse como un proceso de construcción de representaciones significativas y personales de cada estudiante, con sentido común y objetivo y que tenga un espacio real de aplicación o representación en su contexto de actuación profesional.

Se coincide con el autor (Rivera, 2014) cuando establece que el aprendiz sólo aprende cuando encuentra sentido a lo que aprende, es importante resaltar que el docente debe plantearse las estrategias de enseñanza que exige la nueva educación y con ella las propias que se establecen en el modelo educativo institucional, donde se evidencia claramente que no hay espacios para actividades de aprendizaje que desarrolle habilidades como la memoria, la trascripción, la repetición, ya que estas no conducen a un aprendizaje significativo, este se logrará cuando lo que se trabaje como contenido desde la habilidad del pensamiento a desarrollar, bien planteada, logre relacionar de manera coherente, lo que el estudiante tiene como conocimiento previo con lo nuevo que va a adquirir, y en esta relación se analice los aspectos relevantes y preexistentes, donde se construye una nueva teoría o conocimiento dentro de la estructura cognitiva del estudiante.

Partiendo de esta premisa se considera necesario establecer principios básicos que deben asumir nuestros docentes para desarrollar un aprendizaje significativo:

- El conocimiento o las experiencias previas, estas constituyen el punto de partida para el proceso de relación desde la estructura cognitiva del estudiante, dígase conocimiento, contenido anterior o definiciones.

- El rol del docente, como hemos mencionado el docente tiene un papel importante en esta construcción ya que es mediador, facilitador y orientador de las herramientas necesarias para la construcción y relación de los aprendizajes.

- El rol del estudiante, en este caso este debe ser capaz de la autorregulación, autorregulación, autogestión, todos estos procesos como parte de su rol en la construcción y asimilación de aprendizajes con significatividad para ser posteriormente aplicados.

Desde este punto de vista se debe asumir que alcanzamos un aprendizaje significativo cuando el estudiante reconoce que lo aprendido tiene uso, es decir, es de utilidad para relacionarlo con conocimientos previos (Céspedes, Rojas \& Céspedes, 2019). Este aprendizaje estará definido por diversas estrategias y actividades por parte del docente y por parte del estudiante de actitudes que le generen experiencias, le proporcionen cambios y le permitan desde su aplicación la solución de problemas durante su práctica profesional.

\section{Metodología}

Para el desarrollo de la presente investigación de carácter exploratorio se implementaron métodos del nivel teórico y del nivel empírico, tales como: análisis y síntesis, revisión documental, visita a 15 aulas de clases seleccionadas de manera aleatoria, para observación directa y entrevista a los docentes. Estos métodos permitieron, analizar el objeto de investigación desde sus relaciones y cualidades fundamentales, así como recabar información preliminar sobre la necesidad de 


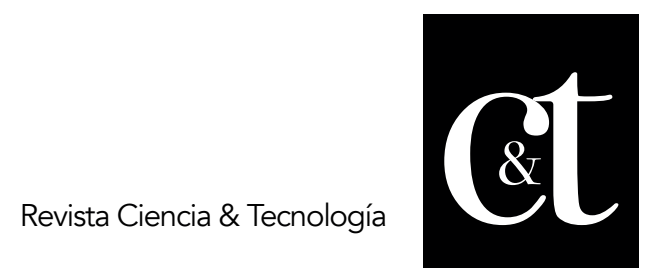

No. 27, 31 de julio de 2020

ISSN impreso: 1390 - 6321

ISSN online: 2661 - 6734

desarrollar un aprendizaje significativo desde nuestras aulas. Para el estudio se contó con la debida autorización por parte del Instituto Superior Tecnológico Almirante Illingworth.

\section{Resultados y discusión}

La revisión de los documentos referentes a la planificación de clases, formatos de planificación de actividades desde los diferentes componentes de aprendizajes, permitió evidenciar que son pocas las actividades que se planifican en función de un aprendizaje significativo, encontrándose que las actividades tanto grupales como individuales no se alinean hacia el resultado de aprendizaje definido. Por su parte, en las visitas a clases se pudo observar de manera directa que las estrategias de enseñanza aplicadas se encaminaban hacia la memorización de conceptualizaciones, transcripción de contenidos y no se lograba una alineación hacia lo propiamente definido en el modelo pedagógico. las estrategias de enseñanza aplicadas se encaminaban hacia la memorización de conceptualizaciones, transcripción de contenidos y no se lograba una alineación hacia lo propiamente definido en el modelo pedagógico.

En las entrevistas a los docentes se puedo evidenciar que poseen conocimiento sobre lo establecido en el modelo pedagógico desde su conceptualización teórica pero no en todas sus clases pueden aplicar estrategias que generen aprendizajes diferentes. Por otro lado, señalan que no logran realizar una caracterización del estudiante por lo que no logran definir el nivel de aprendizaje que poseen ni cuál sería su estilo de aprendizaje, coincidiendo en señalar que planifican una clase para todos los estudiantes sin tener en cuenta cuáles serían las estrategias más apropiadas para llegar a construir un aprendizaje significativo desde las diferencias que existen en cada estudiante hacia sus formas o maneras de aprender. De igual manera, los mismos docentes fueron capaces de proponer algunas de las estrategias que a continuación destacamos.

\section{Propuestas de estrategias para desarrollar aprendizajes significativos}

Es necesario proponer algunas estrategias que permitan al docente asumir y trabajar desde las clases o en las planificaciones de sus actividades desde los diferentes componentes de aprendizaje, entre las cuales se sugiere:

1. Estrategias de enseñanza: estás podrán aplicarse desde los componentes de la clase $o$ en algunas actividades de aprendizajes orientadas.

- Objetivo: es necesario definir el objetivo de la clase (en nuestro caso particular resultado de aprendizaje, que no es más que el objetivo alcanzado) este es el elemento rector del proceso, hacia donde se encamina los restantes componentes de la planificación. De este depende la habilidad a alcanzar, el contenido a trabajar, las actividades de aprendizaje a realizar y las rúbricas para la evaluación.

- Resumen, análisis, mapas conceptuales, organizadores gráficos, analogías: están serán siempre actividades que permitan la vinculación o relación entre los conocimientos previos con los nuevo aprendido, generará un proceso de desarrollo del pensamiento, desde la propia organización y estructuración cognitiva que realice cada estudiante.

- Lectura crítica o analítica: le permitirá al estudiante la relación de lo viejo con lo nuevo, postura y pensamiento crítico reflexivo, criterios propios que sustente el proceso de reflexión. 


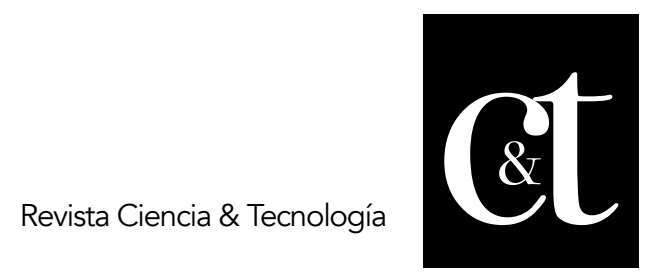

No. 27, 31 de julio de 2020

ISSN impreso: 1390 - 6321

ISSN online: 2661 - 6734

2. Estrategias para la activación o generación de conocimientos previos: se establece en el momento de la anticipación de la clase, donde el docente debe indagar qué conoce ya el estudiante y ubicarlo en el contexto del nuevo conocimiento, desde la orientación del objetivo (resultado de aprendizaje) y los contenidos a abordar.

- Técnica, actividad o recurso inicial que propicie una expectativa en el estudiante hacia lo nuevo por conocer.

- Propiciar situaciones, sistemas de preguntas que generen información sobre conocimientos previos, desde la activación de conocimientos ya adquiridos.

3. Estrategias para la orientación a los estudiantes sobre aspectos significativos de los contenidos de aprendizaje: en este sentido, es importante que el docente pueda vincular con contenidos como conocimientos que el estudiante va alcanzando desde su propia aplicación en la práctica preprofesional, evidenciando la significatividad que estos tienen para su posterior aplicación en contextos reales. Debe centrarse en demostrar la necesidad de que estos aprendizajes sean alcanzados de manera consciente por el estudiante, que el mismo logre reflexionar sobre el por qué debe adquirirlo y en qué momentos estos aprendizajes los podrá aplicar en su práctica profesional real.

4. Estrategias para organización de la nueva información aprendida: bajo esta estrategia el docente debe potenciar esquemas mentales que permitan la organización de la nueva información, el estudiante debe lograr realizar una estructura mental cognitiva que le permita analizar, reflexionar sobre los nuevos aprendizajes y acomodarlos como nuevos conocimientos.

5. Estrategias para promover la relación entre los conocimientos previos y los nuevos adquiridos.

\section{Conclusiones y Recomendaciones}

Constituye una necesidad actual replantearse las formas o estrategias de enseñanza para desarrollar o alcanzar en los estudiantes de la enseñanza superior aprendizajes significativos, ya que estos le permitirán al estudiante desenvolverse como profesionales con habilidades y competencias desarrolladas para según su perfil y campo profesional.

El aprendizaje significativo como resultado del modelo pedagógico constructivista definido por la institución debe ser asumido por todo el personal docente como un proceso de construcción de representaciones significativas y personales de cada estudiante, con sentido común y objetivas y que tenga un espacio real de aplicación o representación en su contexto de actuación profesional (Granja, 2015 y Sandoval, 2019).

Teniendo en cuenta los instrumentos aplicados se puede concluir que es arduo el trabajo que debe continuar realizando el instituto objeto de estudio para que su personal docente establezca estrategias o acciones que permitan desarrollar desde sus clases o actividades de aprendizaje en los diferentes componentes aprendizajes relevantes o significativos, siendo este el objetivo que persigue su actual modelo pedagógico.

Las estrategias de enseñanza, establecidas teniendo en cuenta el objetivo (resultado de aprendizaje) a alcanzar constituyen el eslabón fundamental para el desarrollo de aprendizajes significativos, ya que estas orientan, direccionan el accionar del docente para la obtención de estos fines, es por ello que, deben ser seleccionadas, 


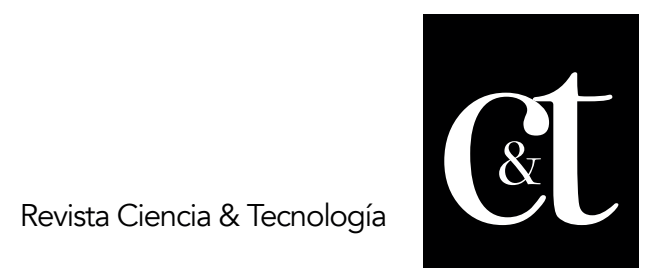

No. 27, 31 de julio de 2020

ISSN impreso: 1390 - 6321

ISSN online: 2661 - 6734

planificadas teniendo en cuenta las características, estilos de aprendizajes y necesidades individuales de cada uno de los estudiantes.

\section{Referencias bibliográficas}

Castellano, D., Reinoso, C., \& García, C. (2014). Colección de proyectos para promover un aprendizaje desarrollador. La habana: Universidad Pedagógica José Varona.

Céspedes, R., Rojas, J. \& Céspedes, Q. (2019). Desarrollo de competencias y aprendizaje Significativo. Balance's, 7(10): 20-24.

González, W. (2018). Una aproximación al aprendizaje desarrollador en la enseñanza superior . Educación Santa María , 11- 26.

Granja, D. O. (2015). El constructivismo como teoría y método de enseñanza. Sophia, Colección de Filosofía de la Educación, 34-46.

López, D. (2011). El proceso de enseñanza aprendizaje desarrollador y su relación con el trabajo metodológico. Revista Científico Pedagógica , 29-38.

Palma-Orozco, R., Garcia-Leyva, E., \& Ruiz-Ledesma, E (2020). Aprendizaje significativo: El caso de la computación, la matemática y la música. Sistemas, cibernética e informática, 17 (1).

Rivera, J. (2014). El aprendizaje significativo y la evaluación de los aprendizajes. Revista de Investigación Educativa, 47 - 59.

Sandoval, V. B. (18 de noviembre de 2019 ). Teorías constructivistas del aprendizaje. Obtenido de Biblioteca digital academia Universidad Académica de Humanismo Cristiano

http://bibliotecadigital.academia.cl/jspui/bitstream/123456789/2682/1/TPE

DIF\%2024.pdf

Varela, M. R. (12 de abril de 2018). Resignificación del modelo pedagógico para el fortalecimiento de las prácticas pedagógicas. Barranquilla, Colombia .

Zambrano, P. S. (2016). La teoría constructivista y su significación para la pedagogía contemporánea . Revista Cinetífica Ciencias Sociales y Políticas, 21- 26. 